\title{
The effect of hippocampal lesions on the development and extinction of a learned taste aversion for a novel food
}

\author{
MICHAEL L. THOMKA and THOMAS S. BROWN \\ DePaul University: Chicago. Illinois 60614
}

\begin{abstract}
The effect of hippocampal lesions on the inhibition of eating was examined within a learned taste aversion paradigm. Rats with hippocampal lesions were tested on their ability to refrain from eating a novel tasting food that had been paired with the internal malaise produced by a .15-M IP injection of lithium chloride. The results showed that the rats with hippocampal lesions did not differ from normal rats on either the development or extinction of the aversion. These results extend previous findings which show that bilateral hippocampal lesions do not interfere with the normal modulation of consummatory behaviors.
\end{abstract}

Attention has recently been given to the effects of hippocampal lesions on learned taste aversions (Best \& Orr, 1973; McGowan, Hankins, \& Garcia, 1972; Murphy \& Brown, 1974). In all of the studies which examined this effect, the taste of a solution was the CS which was paired with a noxious UCS. The results have shown that large hippocampal lesions and ventral hippocampal lesions do not interfere with the acquisition and extinction of a learned taste aversion.

Murphy \& Brown (1974) have interpreted the results of their learned taste aversion study as extending the earlier findings of the effects of hippocampal lesions on passive avoidance tasks which employed a consummatory measure. These passive avoidance experiments (Kaada, Rasmussen, \& Kveim, 1962; Kveim, Setekleiv, \& Kaada, 1964) have shown that rats with hippocampal lesions are capable of refraining from consummatory behavior that leads to aversive consequences (for example, mouth shock). However, the passive avoidance tests, like the learned taste aversion studies, have relied exclusively on drinking as a measure of the ability of a rat with hippocampal lesions to withhold a prepotent consummatory response.

The sole use of drinking as an index of the effect of hippocampal lesions on the inhibition of consummatory behavior may be an important consideration. Several studies (Milgram, 1969; Oliver, Firestone, \& Goodman. 1973) which measured the effect of hippocampal stimulation on both drinking and eating do not support a view of the hippocampus being equally involved with these two behaviors. These data indicate that the hippocampus may exert an inhibitory influence over eating while leaving drinking unaffected. Thus, the exclusive use of tasks which only require the withholding of drinking might produce the potentially misleading notion that rats with hippocampal lesions are

This research was supported in part by the State of Illinois Mental Health Fund Grant 490-13-RD to Dr. Thomas S. Brown. not impaired when required to inhibit any consummatory behavior.

The present study was undertaken to examine the effects of hippocampal lesions on the inhibition of eating. Rats with hippocampal lesions were tested on their ability to keep from eating a novel food which served as a CS within the learned taste aversion paradigm.

\section{METHOD}

\section{Subjects}

The subjects were 18 male Sprague-Dawley rats that averaged $356 \mathrm{~g}$ in weight. The subjects were randomly divided into three equal groups: hippocampal ablates (Group $\mathrm{H})$, cortical ablates (Group $\mathrm{C}$ ), and sham-operated control rats (Group $\mathbf{N}$ ).

\section{Surgery}

All subjects were anesthetized with IP injections of sodium pentobarbital $(50 \mathrm{mg} / \mathrm{ml})$ in proportion to their body weight $(35 \mathrm{mg} / \mathrm{kg})$. An additional injection of $.1 \mathrm{cc}$ atropine sulfate $(.4 \mathrm{mg} / \mathrm{ml})$ was similarly administered to facilitate recovery from anesthesia.

Each subject was placed in a head holder and had its scalp incised and the temporal muscles retracted. For those animals in Groups $\mathrm{C}$ and $\mathrm{H}$, bilateral craniotomy was performed between the bregma and lamda sutures. The exposed dura was then incised, and the cortex overlying the hippocampus was removed by aspiration. This was accomplished under visual guidance. Subjects in Group $\mathrm{H}$ additionally received aspiration of the hippocampus as completely as possible without damaging the underlying thalamus. All surgical wounds were packed with Gelfoam, and the temporal muscles were then sutured closed.

\section{Procedure}

Subjects were given a 2-week recovery period during which food and water were always available in the home cage. At the end of the postoperative recovery period, the experiment was begun. Each day was initiated by removing the subjects from their home cages, weighing them, and placing them in individual plastic testing cages. These cages measured $48 \mathrm{~cm}$ long, $26 \mathrm{~cm}$ wide, and $16 \mathrm{~cm}$ high. A metal grid served as a cover.

During Days 1-8, $30 \mathrm{~g}$ of Teklad Mouse and Rat Diet were placed at one end of the testing cages. Each subject was given 1-h 

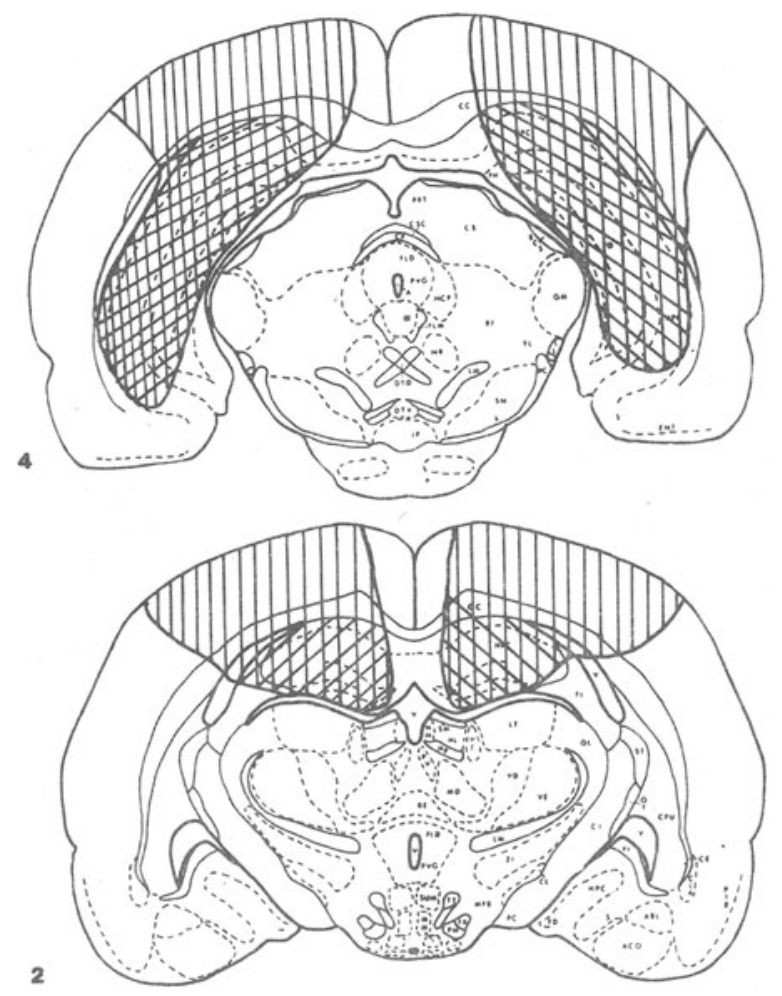

Figure 1. Representative neocortical (striped areas) and hippocampal lesions (cross-hatched areas) superimposed on plates from Pellegrino and Cushman (1967) atlas. (Numbers refer to millimeters posterior to bregma.)

access to the food. No subject was observed to eat more than $30 \mathrm{~g}$ of food during this time. The amount of food eaten during a 1-h feeding session was determined by subtracting the amount of food left in the cage from the initial 30-g ration. The daily feeding session provided the only access a subject had to food. Although water was available in the home cages, it was never available in the testing cages.

Day 9 was the treatment day. On this day, the Teklad pellets were removed from the testing cages, and subjects were given 1-h access to honey-flavored Teklad pellets. This novel food was made by dipping individual Teklad pellets into honey. The pellets were then placed in a paraffin oven until the honey coating became dry. Pilot work showed that rats will readily eat this food. Fifteen minutes after being returned to the home cage, each animal was given an IP injection of a .15-M solution of lithium chloride $(\mathrm{LiCl})$ dissolved in physiological saline. All animals received a $2 \%$ body weight dose of the $\mathrm{LiCl}$.

On Days $10,11,13$, and 15 , subjects once again had access to the standard Teklad diet in the testing cages. These days separated 3 test days (Days 12,14, and 16) during which honey-flavored pellets were present. The alternate access to the standard Teklad diet allowed animals to regain any weight that might have been lost due to avoiding the honey-flavored pellets. The amount of honey-flavored food eaten on the test days provided a measure of the extent of the aversion and the rate of its extinction.

\section{RESULTS}

\section{Histological}

Figure 1 shows representative samples of hippocampal and cortical lesions. The hippocampal lesions tended to be large and uniform in extent. Approximately $80 \%$ of the hippocampus was damaged with some variation resulting from different amounts of the posteroventral tip of the hippocampus remaining intact. In all cases, the septum and cingulate gyrus were spared. Occasionally, small portions of the dorsal thalamus was ablated. However, this damage was unilateral and not correlated with behavior. Animals in the cortical control group had lesions restricted to the cortex directly overlying the hippocampus. In some instances, slight hippocampal damage was observed, but this also was not correlated with behavior. The amount of cortical damage for animals in both the hippocampal group and the cortical group was similar.

\section{Behavioral}

Table 1 shows the mean amount of food eaten on the treatment day (Day 9) and each of the 3 test days (Days 12,14 , and 16). It can be seen that all three groups initially reduced their consumption of the honey-flavored pellets after it was paired with the noxious effects of $\mathrm{LiCl}$. T tests for matched pairs revealed significant decreases in the mean amount of food eaten on the 1 st test day (Day 12 ) relative to the treatment day for Group $\mathrm{H}(\mathrm{t}=8.45, \mathrm{df}=5, \mathrm{p}<.001)$, Group C $(\mathrm{t}=10.6, \mathrm{df}=5, \mathrm{p}<.001)$, and Group $\mathrm{N}$ $(t=6.97, d f=5, p<.001)$. Thus, the pairing of $\mathrm{LiCl}$ with the taste of a novel food produced a significant taste aversion in each group.

The course and relative magnitude of the taste aversion over the 3 test days is shown in Figure 2. In this figure, the aversion is expressed as a percentage of the amount of food eaten on the treatment day so that the lower percentages indicate greater aversions. This measure was chosen because the animals in Group $\mathrm{H}$ ate significantly more of the honey-flavored pellets on the treatment day than did the animals in Group $\mathrm{C}(\mathrm{t}=3.22$, $\mathrm{df}=10, \mathrm{p}<.01)$. Since the amount of food eaten was converted to a percentage score for each test day, Kruskal-Wallis one-way analyses of variance were performed on the obtained percentages. Individual comparisons between groups means, taken two at a time, were done using Mann-Whitney U tests, as suggested by Winer (1971, p. 849).

Table 1

Mean Amount of Food Eaten in Grams by Each Group as a Function of Treatment Day (Day 9) and Three Test Days (Days 12, 14, and 16)

\begin{tabular}{rrrl}
\hline Day & $\mathrm{H}$ & $\mathrm{N}$ & $\mathrm{C}$ \\
\hline 9 & 12.1 & 9.9 & 8.3 \\
12 & 6.5 & 6.2 & 2.8 \\
14 & 11.6 & 10.1 & 7.4 \\
16 & 11.3 & 10.2 & 9.7 \\
\hline
\end{tabular}

Note-Brackets indicate significant differences between means at .001 level. 
Table 2 shows the mean percentages of food eaten for each subject and each group on the 3 test days. As can be seen from Table 2, on Day 12, subjects consumed a mean of $50.7 \%$ of what they had eaten on the treatment day. Comparisons of individual group means for Day 12 [after obtaining an overall significant group effect $(\mathrm{H}=33.03, \mathrm{df}=2, \mathrm{p}<.005)]$ showed that rats in Group C displayed a significantly $(\mathrm{p}<.05)$ greater aversion (32.6\%) than animals in either Group H (56.3\%) or Group N (63.3\%). However, Group H and Group N did not differ on the magnitude of the aversion on this day $(\mathrm{p}>.05)$.

Subjects in all groups began to show extinction for the aversion by the second test day (Day 14). As can be seen from Table 2, subjects were eating $93.9 \%$ of what they had previously consumed on the treatment day. Analysis of the mean percentages did not reveal any between group differences for the day $((\mathrm{H}=1.15 \mathrm{df}=2$, $\mathrm{p}>.05$ ).

By Day 16, the aversion was fully extinguished with animals returning to treatment day levels of consumption (104.9\%). Significant differences between groups were also obtained $(\mathrm{H}=15.55, \mathrm{df}=2, \mathrm{p}<.01)$. This difference reflected the animals with cortical lesions eating a relatively greater $(p<.05)$ percentage $(119.5 \%)$ of food than the rats with hippocampal lesions $(92 \%)$ or the normal rats $(103.2 \%)$. Animals in Group $\mathrm{H}$ and Group, $\mathrm{N}$ had similar percentages of food intake $(\mathrm{p}>.05)$.

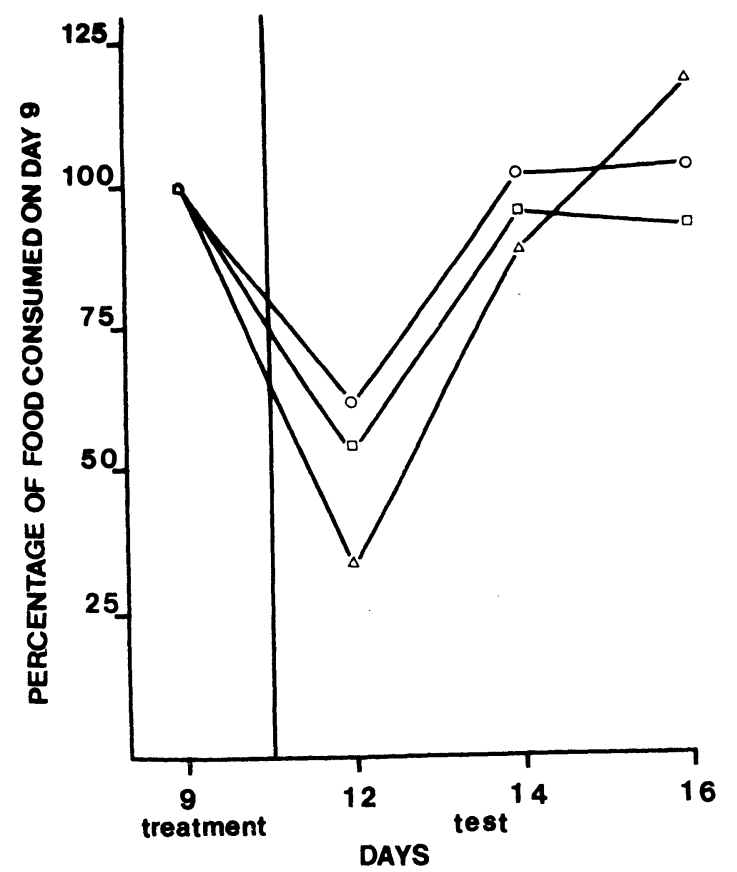

Figure 2. Course of the aversion over 3 test days (Days 12, 14, and 16) expressed as the percentage of food eaten on the treatment day (Day 9).
Table 2

Mean Percentage of Food Eaten Relative to Treatment Day (Day 9) for Each Subject on Three Test Days (Days 12, 14, and 16)

\begin{tabular}{|c|c|c|c|c|}
\hline \multirow{2}{*}{\multicolumn{2}{|c|}{$\begin{array}{l}\text { 苛 Sub- } \\
\text { Sj ject }\end{array}$}} & \multicolumn{3}{|c|}{ Mean Percentage } \\
\hline & & Day 12 & Day 14 & Day 16 \\
\hline $\mathrm{H}$ & $\begin{array}{l}\text { H-1 } \\
\text { H-2 } \\
\text { H-3 } \\
\text { H-4 } \\
\text { H-5 } \\
\text { H-6 }\end{array}$ & $\begin{array}{l}66 \\
67 \\
36 \\
66 \\
56 \\
47\end{array}$ & $\begin{array}{r}127 \\
109 \\
68 \\
77 \\
108 \\
81\end{array}$ & $\begin{array}{r}116 \\
94 \\
72 \\
91 \\
105 \\
74\end{array}$ \\
\hline & Mean & 56.3 & 95.0 & 92.0 \\
\hline $\mathrm{C}$ & $\begin{array}{l}\text { C-1 } \\
C-2 \\
\text { C-3 } \\
\text { C-4 } \\
\text { C-5 } \\
C-6\end{array}$ & $\begin{array}{l}26 \\
11 \\
29 \\
43 \\
36 \\
51\end{array}$ & $\begin{array}{r}91 \\
93 \\
74 \\
100 \\
81 \\
98\end{array}$ & $\begin{array}{l}161 \\
121 \\
107 \\
107 \\
114 \\
107\end{array}$ \\
\hline & Mean & 32.6 & 88.8 & 119.5 \\
\hline $\mathrm{N}$ & $\begin{array}{l}\text { N-1 } \\
\text { N-2 } \\
\text { N-3 } \\
\text { N-4 } \\
\text { N-5 } \\
\text { N-6 }\end{array}$ & $\begin{array}{l}50 \\
66 \\
58 \\
64 \\
79 \\
63\end{array}$ & $\begin{array}{r}105 \\
66 \\
113 \\
99 \\
88 \\
117\end{array}$ & $\begin{array}{r}103 \\
89 \\
123 \\
102 \\
99 \\
103\end{array}$ \\
\hline & Mean & 63.3 & 98.0 & 103.2 \\
\hline Total & Mean & 50.7 & 93.9 & 104.9 \\
\hline
\end{tabular}

\section{DISCUSSION}

The main result of the present experiment is that bilateral lesioning of the hippocampus does not disrupt the acquisition or extinction of a learned taste aversion for a novel food. At each point tested, the performance of rats with hippocampal lesions did not differ from that of the normal animals. In contrast, the animals with cortical lesions developed a greater aversion than did the animals in either the $\mathrm{H}$ or $\mathrm{C}$ groups. This is an unexpected finding since earlier work (Murphy \& Brown, 1974) which employed comparable cortical lesions did not find any difference between rats with cortical lesions and normal rats on the extent of a learned taste aversion for a novel sucrose solution. It is interesting to note, however, that Hankins, Garcia, \& Rusiniak (1974) have shown that posterior cortical lesions, which appear similar to the cortical lesions in the present study, produce a slightly greater aversion for a saccharin solution than that observed in normal animals. It is thus uncertain at present what effect a large cortical lesion has on the development of a learned taste aversion.

Regarding the effect of hippocampal lesions on learned taste aversion, the results clearly demonstrate that rats with hippocampal lesions are capable of associating the taste of a novel food with a noxious UCS. Garcia, McGowan, Ervin, and Koelling (1968) have previously demonstrated this for the normal rat. It also 
appears that the rat with hippocampal lesions can as readily associate a novel food with induced sickness as it can a novel solution. This is indicated by the fact that the present procedures produced a $44 \%$ reduction in eating the novel food on the part of Group $\mathrm{H}$ animals which is similar to the magnitude of previous taste aversions for novel liquids. McGowan, Hankins, \& Garcia (1972) found that rats with ventral hippocampal lesions reduced their intake of a saccharin solution by approximately $40 \%$ after it was paired with internal malaise and that rats with dorsal hippocampal lesions reduced their intake of the same solution by approximately $30 \%$. In addition, Murphy and Brown (1974) demonstrated a learned taste aversion to sucrose with rats with bilateral hippocampal lesions reducing their intake by $60 \%$.

The present experiment does not confirm expectations based on the differential effect of stimulation of the hippocampus on eating and drinking (Milgram, 1969; Oliver, Firestone, \& Goodman, 1973). Several procedural differences may account for this disparity. The present study used hungry animals given limited amounts of food while the stimulation studies employed animals on ad-lib regimes. Stimulation was also applied only to small areas of the hippocampus at any one time. The present study investigated a large area of hippocampal involvement. Finally, stimulation of the hippocampus may have affected other areas of the brain which were not influenced after aspiration of the hippocampus.

The similarity between the learned taste aversion and passive avoidance designs has already been pointed out (Best \& Orr, 1973; Murphy \& Brown, 1974). The present results indicate that rats with hippocampal lesions are capable of inhibiting eating on what is essentially a passive avoidance task.

It has been observed (Murphy \& Brown, 1970; Murphy \& Brown, 1974) that rats with hippocampal lesions are able to inhibit drinking but not locomotion on passive avoidance tasks. This has led to a reappraisal of the proposed function (Douglas, 1967; Kimble, 1968) of the hippocampus as a general inhibitor of ongoing behavior. Murphy and Brown (1970) speculate that the hippocampus exerts inhibitory control over locomotor behavior (walking or running) while leaving unaffected those behaviors which are consummatory in nature (drinking). The findings of this experiment add to the evidence which support the hypothesis of Murphy and Brown by demonstrating that rats with hippocampal lesions are also not impaired when challenged. with inhibiting eating.

\section{REFERENCES}

Best, P. J., \& Orr, J. Effects of hippocampal lesions on passive avoidance and taste aversion conditioning. Phy siology and Behavior, 1973, 10, 193-196.

Douglas, R. J. The hippocampus and behavior. Psychological Bulletin, 1967, 67, 416-442.

Garcia, J., McGowan, B. K., Ervin, F. R., \& Koelling, R. A. Cues: Their relative effectiveness as a function of the reinforcer. Science, 1968, 160, 794-795.

Hankins, W. G., Garcia, J., \& Rusiniak, K. W. Cortical lesions: flavor illness and noise-shock conditioning. Behavioral Biology, 1974, 10, 173-181.

Kaada, B. R., Rasmussen, E. W., \& Kveim, O. Impaired acquisition of passive avoidance by subcallosal, septal, hypothalamic and insular lesions in rats. Journal of Comparative and Physiological Psychology, 1962, 55, 661-670.

Kimble D. P. Hippocampus and internal inhibition. Psy chological Bulletin, 1968, 70, 285-295.

Kveim, O., Setekleiv, J., \& Kaada, B. R. Differential effects of hippocampal lesions on maze and passive avoidance learning in rats. Experimental Neurology, 1964, 9, 59-72.

McGowan, B. K., Hankins, W. G., \& Garcia, J. Limbic lesions and control of the internal and external environment. Behavioral Biology, 1972, 7, 841-852.

Milgram, N. W. Effect of hippocampal stimulation on feeding in the rat. Phy siology and Behavior, 1969, 4, 665-670.

Murphy, H. M.. \& Brown, T. S. Effects of hippocampal lesions on simple and preferential consummatory behavior in the rat. Joumal of Comparative and Physiological Psychology, 1970, 72, 404-415.

Murphy, L. R., \& Brown, T.S. Hippocampal lesions and learned taste aversion. Phy siological Psy chology, 1974, 2, 60-64.

Oliver, D. L., Firestone, M. I., \& Goodman, E. D. Hippocampal stimulation rebound effects in hamsters. Physiology and Behavior, 1973, 10, 23-28.

Winer, B. J. Statistical principles in experimental design. New York: McGraw-Hill, 1971.

(Received for publication January 29, 1975; revision accepted March 17, 1975.) 